\title{
IKNO, a user facility for coherent terahertz and UV synchrotron radiation
}

\section{Fernando Sannibale, ${ }^{a^{*}}$ Augusto Marcelli ${ }^{\mathrm{b}}$ and Plinio Innocenzi ${ }^{\mathrm{c}}$}

\author{
a'Lawrence Berkeley National Laboratory, Berkeley, CA 94720, USA, ${ }^{b}$ Istituto Nazionale di Fisica Nucleare \\ - Laboratori Nazionali di Frascati, 00044 Frascati, Italy, and ' University of Sassari, 074041 Alghero, Italy. \\ E-mail: fsannibale@Ibl.gov
}

Synopsis IKNO, a proposal for a multi-user synchrotron radiation source optimised for the generation of coherent synchrotron radiation in the terahertz frequency range with many orders of magnitude higher photon flux than in existing sources is presented. Additionally, the incoherent radiation spectrum of IKNO extends up to the VUV frequencies with flux comparable with that of a 3rd generation synchrotron light source.

\begin{abstract}
IKNO (Innovation and KNOwledge) is a proposal for a multi-user facility based on an electron storage ring optimised for the generation of coherent synchrotron radiation (CSR) in the terahertz frequency range, and of broadband incoherent synchrotron radiation (SR) ranging from the IR to the VUV. IKNO can be operated in an ultrastable CSR mode with photon flux in the terahertz frequency region up to nine orders of magnitude higher than in existing 3rd generation light sources. Simultaneously to the CSR operation, broadband incoherent SR up to VUV frequencies is available at the beamline ports. The main characteristics of the IKNO storage and its performance in terms of CSR and incoherent SR are described in this paper. The proposed location for the infrastructure facility is in Sardinia, Italy.
\end{abstract}

Keywords: synchrotron radiation; synchrotron radiation source; terahertz; coherent synchrotron radiation; UV.

\section{Introduction}

The interest in science using radiation in the terahertz region is steadily increasing (Sherwin et al., 2004) (THz network: http://www.thznetwork.org). The availability of high power, short-pulse radiation in this low energy range is very important for studying collective excitations in solids, protein conformational dynamics, superconductor bandgaps and electronic and magnetic scattering. Unluckily, generating radiation of significant intensity in this frequency range, between microwaves and infrared (IR), is not straightforward and this region is often referred to as the "terahertz gap". Many fields are largely unexplored due to the lack of intense sources, and a facility capable of providing short pulsed and high average power radiation in this spectral region may offer unique opportunities for probing chemical, physical, biological processes and support high-tech and space researches and applications.

In the last years, there has been significant progress in the understanding of CSR in electron storage rings. A model accounting for the CSR terahertz random bursts observed at high single bunch current in many rings was proposed (Stupakov \& Heifets, 2002; Venturini \& Warnock, 2002) and experimentally verified (Byrd et al., 2002;

*This work was supported by the U.S. Department of Energy under Contract No. DE-AC02-05CH11231. 
Abo-Bakr et al., 2003). Stable CSR was produced for the first time in a storage ring (Abo-Bakr et al.,2002; Abo-Bakr et al., 2003) and a theoretical model accounting for the observations was developed (Sannibale et al., 2004; Sannibale et al., 2004, ICFA). A new technique based on the energy modulation of a fraction of the electron beam by a fs laser pulse and that can generate intense sub-ps pulses of THz CSR has been developed and characterized (Zholents \& Zolotorev, 1996; Byrd et al., 2005; Holldack et al., 2005; Holldack et al., 2006; Byrd et al., 2006; Heifets et al., 2006; Byrd et al., 2006, Phys. Rev. Lett. 97; Bielawski et al., 2008). In this scheme, the intrinsically synchronous sub-ps pulses of CSR and of the modulating laser allow for high time resolution pump and probe experiments. CIRCE (Barry et al., 2002; Byrd et al., 2004; Byrd et al., 2005, CERN Courier), a ring-based source completely optimised for the generation of coherent terahertz synchrotron radiation and that exploits the full complement of the CSR production mechanisms mentioned above was proposed at the Lawrence Berkeley National Laboratory (Barry et al., 2002; Byrd et al., 2004; Byrd et al., 2005, CERN Courier).

$\mathrm{THz}$ CSR has been also generated in schemes using linear accelerators. Remarkable examples can be found in references (Carr et al., 2002; Shen et al., 2007). Additionally, most of the so-called future $4^{\text {th }}$ generation light sources (sources based on energy recovery linacs and free electron lasers) include one or more ports for $\mathrm{THz} \mathrm{CSR}$ in their layout. In general, storage ring based sources show a potentially higher stability and are capable to accommodate a larger number of users, while linac-based sources can potentially achieve a higher peak power in the single radiation pulse. Overall, the two schemes show significant complementary characteristics that can cover the whole spectrum of science in the field.

We are proposing IKNO (acronym for Innovation and KNOwledge but also the ancient name of the Sardinia island in Italy) a ring-based multi user facility optimized for the generation of THz CSR to be built in Sardinia. IKNO is a more compact version of CIRCE and is capable of a calculated photon flux exceeding by more than nine orders of magnitude the one of existing synchrotron light sources. In addition to the THz CSR mode, IKNO allows for dedicated and parasitic modes of operation for the generation of broadband incoherent SR in a wide spectral range and with flux comparable to the ones in modern 3rd generation light sources.

\section{The IKNO storage ring}

Table 1 shows the main parameters for the IKNO electron storage ring.

The lattice includes six Double Bend (DB) cells with two 30 deg bending magnets and $3.1 \mathrm{~m}$ long straight sections between the arcs. The linear dynamics of the electrons in the ring is defined by the magnetic field settings in the cell and can be described by the so-called optical functions. The DB optical functions for IKNO are shown in Figure 1.

The bending magnets (B) include a gradient of $\sim 2.1 \mathrm{~T} / \mathrm{m}$ at $600 \mathrm{MeV}$ that provides the focusing in the vertical plane. Three families of quadrupole magnets $\left(\mathrm{Q}_{1}, \mathrm{Q}_{2}, \mathrm{Q}_{3}\right)$ all focusing in the horizontal plane define the transverse tunes (frequency of the electron oscillation in the transverse plane) and allow for the control of the first order momentum compaction (longitudinal dispersion term). This task is accomplished by changing the value of the 
transverse dispersion $\eta_{x}$ on the straights while keeping constant the transverse tunes. In this way the first order momentum compaction of the ring can be continuously tuned from positive to negative values (Fig.1 shows the lattice with a first order momentum compaction of 0.0013). This capability is of extreme importance because the CSR performance of the ring strongly depends on the control of the momentum compaction and of its higher order terms. Three families of sextupole magnets $\left(\mathrm{S}_{1}, \mathrm{~S}_{2}, \mathrm{~S}_{3}\right)$ permit chromaticity correction (transverse tune dependence on the particle energy) and tuning of the second order term of the momentum compaction. Finally, additional coils in each of the quadrupole magnets can generate an octupolar field component for the direct control of the third order term of the momentum compaction.

Table 1 IKNO Ring main parameters.

\begin{tabular}{|l|l|l|l|}
\hline Energy [MeV] & 600 & Ring Length [m] & 60 \\
\hline $\begin{array}{l}\text { Natural Emittance } \\
{[\mathrm{nm}]}\end{array}$ & 41 & $\begin{array}{l}\text { Relative Energy Spread } \\
(\mathrm{rms})\end{array}$ & $0.46 \times 10^{-3}$ \\
\hline RF freq. [GHz] & 1.5 & RF Voltage [MV] & 1.2 \\
\hline Harm. Number & 300 & Bend Radius [m] & 1.335 \\
\hline $\begin{array}{l}\text { Energy lost/turn } \\
\text { (incoh. SR)[keV] }\end{array}$ & 8.6 & $\begin{array}{l}\text { Damping times } \\
\text { (Hor./Vert./Long.) }[\mathrm{ms}]\end{array}$ & $\begin{array}{l}24.5 / 28.0 / \\
15.0\end{array}$ \\
\hline Hor. betatron tune & 5.15 & Vertical betatron tune & 4.2 \\
\hline Hor. chromaticity & -7.1 & Vertical chromaticity & -21.75 \\
\hline
\end{tabular}

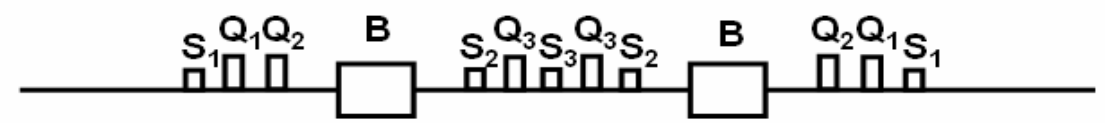

$I K N O, 60 \mathrm{~m}, D B$ Cell, $k($ bend $)=-1.4-600 \mathrm{MeV}$

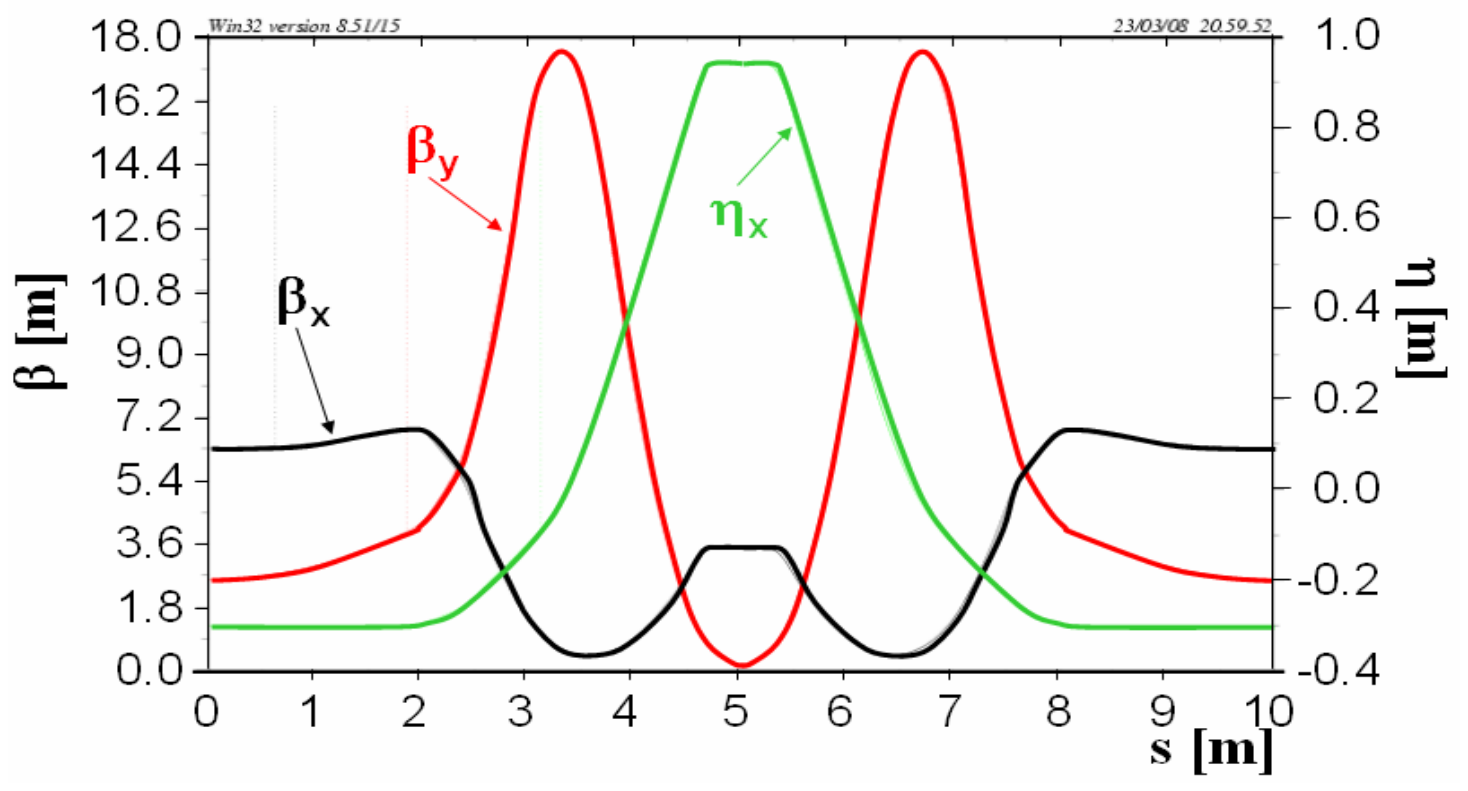

Figure 1 IKNO DB cell optical functions. 
Downstream of each of the bending magnets a drift of $\sim 0.5 \mathrm{~m}$ allows for the stay clear area required by the beamline front-ends. In the present layout, such front-ends include a $45 \mathrm{deg}$ in vacuum mirror that reflects the synchrotron radiation upwards and through a viewport that separates the storage ring vacuum from the one of the beamlines.

Transverse tunes and natural chromaticities are shown in Table 1. The tunes for the horizontal and vertical planes have been chosen exploiting the results of calculations previously done for CIRCE that showed a good dynamic aperture (acceptance for large orbit deviation) in this tune region, allowing for an easy injection of the electron beam in the ring and for a good beam lifetime (Nishimura et al., 2004).

The maximum magnet strengths required for tuning the ring among a variety of lattices and chromaticity values are always smaller than $5 \mathrm{~m}^{-2}$ for the $20 \mathrm{~cm}$ long quadrupoles, and $50 \mathrm{~m}^{-3}$ for the $15 \mathrm{~cm}$ long sextupoles. Those strengths at $600 \mathrm{MeV}$ electron energy, correspond to the modest gradient values of $10 \mathrm{~T} / \mathrm{m}$ and $100 \mathrm{~T} / \mathrm{m}^{2}$ for the quadrupole and the sextupole respectively (readily achievable with conventional technology).

Requirements on the IKNO beam emittance (phase space volume occupied by the electron beam) are quite relaxed because the photon source size is diffraction limited in the terahertz region. This situation allows for a relatively large natural emittance and coupling (ratio between vertical and horizontal emittance) values with a consequently lower beam particle density that reduces electron-electron scattering in the electron bunch (Touschek effect) with a beneficial impact on the beam lifetime.

The RF voltage necessary for restoring the energy radiated by the particles $(1.2 \mathrm{MV}$ at $1.5 \mathrm{GHz})$ can be obtained by multiple normal-conducting cavities or by a single super-conducting cavity.

The vacuum chamber in the dipole magnets and the first in-vacuum mirror must be designed with a large vertical acceptance for matching the large emission angle of the terahertz synchrotron radiation for an efficient extraction of the radiation itself. We are planning to use the scheme proposed by CIRCE (Barry et al., 2002; Byrd et al., 2004; Byrd et al., 2005, CERN Courier; Nishimura et al., 2004; De Santis et al., 2003) where each of the dipoles can accommodate up to three beamline front ends with the very large horizontal and vertical acceptances of $100 \mathrm{mrad}$ and $140 \mathrm{mrad}$ respectively, for a potential of 36 total bending magnets beamlines.

Two of the $3.1 \mathrm{~m}$ straight sections are occupied by RF system and by the injection. The remaining four can be advantageously used for insertion device beamlines. A few appealing possibilities are described in section 4.

\section{Ultra-stable terahertz CSR mode.}

IKNO's principal mode of operation is the ultra-stable CSR mode described in references (Sannibale et al., 2004; Sannibale et al., 2004, ICFA). This mode allows for the simultaneous combination of high and stable power, features of extreme importance for most of the experiments using terahertz radiation.

Figure 2 shows the impressive flux of IKNO in the $\mathrm{THz}$ region, calculated for three examples of ultra-stable settings, and compared with the flux of a 40 mrad acceptance beamline at the Advanced Light Source in Berkeley, a 
typical 3rd generation light source. The up to nine orders of magnitude higher flux in the $\mathrm{THz}$ region is clearly visible.

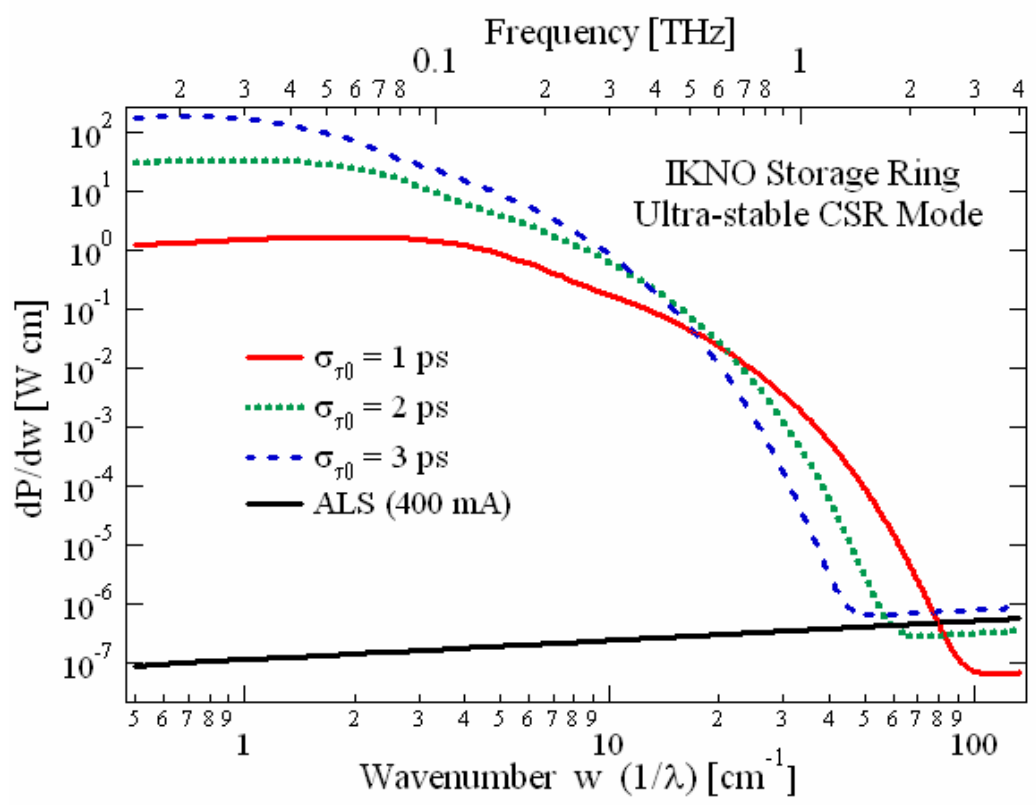

Figure 2 IKNO photon flux in the ultra-stable mode for the single bending magnet port with 100 mrad horizontal acceptance.

In the ultra-stable mode of operation, the fields associated with the synchrotron radiation emitted by the electrons in the bend magnets, generate a stable distortion of the bunch longitudinal distribution from the usual Gaussian to a sawtooth-like shape with a sharp leading edge (Bane et al., 1995). Such a distribution radiates CSR at frequencies much higher than for the case of a pure gaussian bunch with same rms length.

For a steady and stable flux of photons, fundamental for most of the $\mathrm{THz}$ experiments, the current per bunch must be maintained below the microbunching instability threshold (MBI) (Stupakov \& Heifets, 2002; Sannibale et al., 2004; Sannibale et al., 2004, ICFA) for avoiding the strong CSR THz random bursts associated with the instability.

Table 2 IKNO parameters for the three different ultra-stable settings shown in Fig. 2. Power and energy values are calculated for the bending magnet horizontal acceptance of $100 \mathrm{mrad}$ and integrated from 1 to $100 \mathrm{~cm}^{-1}$.

\begin{tabular}{|l|l|l|l|l|l|l|l|}
\hline & $\begin{array}{l}\text { Natural } \\
\text { bunch } \\
\text { length } \\
(\mathrm{rms})[\mathrm{ps}]\end{array}$ & $\begin{array}{l}\text { Momentum } \\
\text { Compaction }\end{array}$ & $\begin{array}{l}\text { Total } \\
\text { stored } \\
\text { current } \\
{[\mathrm{mA}]}\end{array}$ & $\begin{array}{l}\text { Current } \\
\text { per } \\
\text { bunch } \\
{[\mu \mathrm{A}]}\end{array}$ & $\begin{array}{l}\text { Total } \\
\text { CSR } \\
\text { power } \\
{[\mathrm{W}]}\end{array}$ & $\begin{array}{l}\text { Pulse } \\
\text { peak } \\
\text { power } \\
{[\mathrm{kW}]}\end{array}$ & $\begin{array}{l}\text { per } \\
\text { pulse }\end{array}$ \\
\hline SET 1 & 1.0 & $4.5 \times 10^{-4}$ & 29.7 & 99.0 & 8.6 & -2.3 & 5.7 \\
\hline SET 2 & 2.0 & $1.8 \times 10^{-3}$ & 151 & 502 & 73.5 & -9.8 & 49 \\
\hline SET 3 & 3.0 & $4.0 \times 10^{-3}$ & 380 & 1265 & 219 & -19 & 146 \\
\hline
\end{tabular}


Table 2 shows the IKNO main parameters and performance for the three different spectra shown in Fig. 2. In the ultra-stable SET 1, the momentum compaction is tuned to a lower value to generate a shorter natural bunch length. This reduces the MBI current threshold and thus the CSR intensity, but extends the CSR spectrum towards higher frequencies. In SET 3, a larger momentum compaction increases the threshold for the burst instability allowing for more current per bunch and more CSR power at the cost of a reduced bandwidth. Finally, SET 2 represents an example of a situation in between the two previous modes.

Electromagnetic shielding effects due to the conductive vacuum chamber can jeopardize the CSR performance of a ring-based source. The curves in Fig.2 have been calculated assuming no shielding in the dipole vacuum chamber. This situation can be realistically achieved by a proper choice of the bending magnet vacuum chamber vertical halfheight $h$ according to the criterion (Murphy et al., 1997; Sannibale et al., 2004; Sannibale et al., 2004, ICFA):

$$
\frac{\sigma_{z 0}}{\lambda_{\text {Cutoff }}}=\sigma_{z 0} \frac{\rho^{1 / 2}}{2 h^{3 / 2}}<0.2
$$

where $\sigma_{z 0}$ is the natural bunch length, $\rho$ the bending radius and $\lambda_{\text {Cutoff }}$ the dipole vacuum chamber cutoff wavelength. The SET 3 ultra-stable mode of operation for IKNO has the longest bunches (3 ps) and represents the worst case from the chamber shielding point of view. A bending magnet vacuum chamber half-height of at least 1.9 $\mathrm{cm}$ is required for making the shielding effects negligible for this case. Conservatively, we assume $h=2.5 \mathrm{~cm}$ that gives a long wavelength cutoff $\lambda_{\text {Cutoff }} \sim 7 \mathrm{~mm}$ or equivalently a $f_{\text {Cutoff }} \sim 0.04 \mathrm{THz}$.

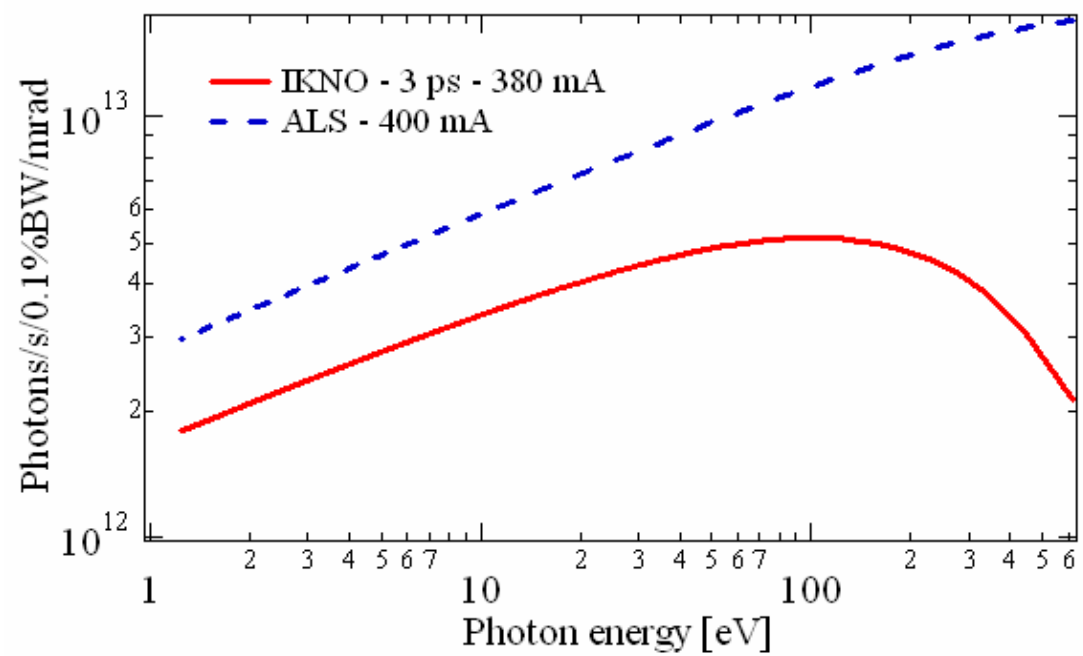

Figure 3 Bend magnet synchrotron radiation high frequency spectrum for IKNO compared with the one for the ALS.

It has been shown that in addition to the shielding effects, also the resistive wall impedance of the ring must be contained in order to preserve the CSR performance (Sannibale et al., 2004; Sannibale et al., 2004, ICFA). For this reason, the use of a high conductivity material such as aluminum for the vacuum chamber is preferable. 
The red solid line in Fig. 3 shows the incoherent higher frequency part of the spectrum of the radiation from a bend magnet when IKNO is operated in the 3 ps ultra-stable mode of operation. The comparison with the ALS case (dashed blue line) shows that the photon flux up to energies of $50 \mathrm{eV}$ is only $\sim 2$ times smaller than the one of the much higher electron energy (and much more expensive) ALS ring.

\section{Exploiting the straight sections.}

Insertion devices such as undulators optimised for the generation of radiation in the UV-VUV range can be naturally inserted in the $3.1 \mathrm{~m}$ IKNO straight sections. Figure 4 shows an example of the significant photon flux obtainable by a $2.72 \mathrm{~m}$ long, $8 \mathrm{~cm}$ period device when the ring is operated in the $3 \mathrm{ps}$ ultra-stable mode.

In one of the straight sections, is also possible to include an undulator magnet to allow fs laser modulation of the electron beam (Zholents \& Zolotorev, 1996). In this scheme, a short laser pulse in the visible or in the near IR is propagated together with the electron beam in the undulator and modulates the energy of the electrons in a short slice ( $\sim 100 \mathrm{fs}$ ) of the bunch. Due to the ring longitudinal dispersion (nonzero momentum compaction), the particles in the slice are displaced and create a density modulation in the bunch longitudinal distribution when the beam propagates along the storage ring. Such a modulation radiates an intense short pulse of THz CSR which is intrinsically synchronous with the slicing laser pulse. Calculations done for CIRCE (Barry et al., 2002) showed that if properly tuned, this scheme is capable to generate sub-ps THz CSR pulses with energy per pulse approaching the $10 \mu \mathrm{J}$ and with spectrum extending to up few tens of $\mathrm{THz}$. The repetition rate in this technique is limited by the power available in the modulating laser and can achieve, with the present laser technology and for acceptable costs, tens of kHz. The simultaneous presence of the two synchronous pulses (the THz CSR one and the visible one from the modulating laser) opens the possibility for pump and probe experiments with sub-ps resolution.

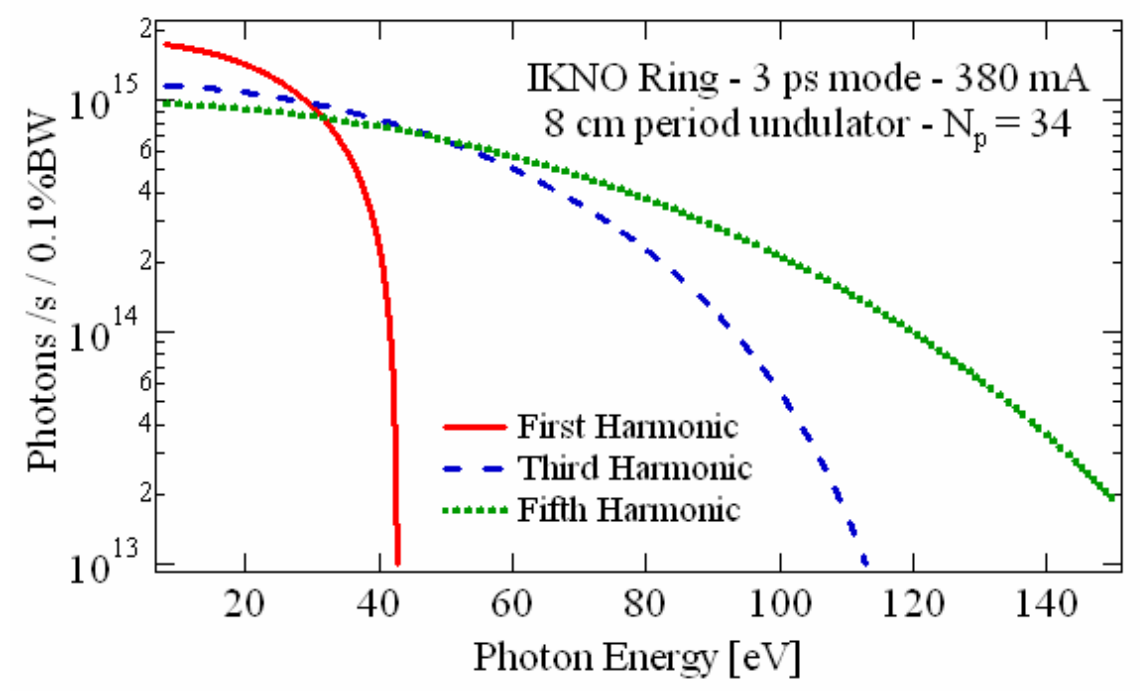

Figure 4 Example of undulator photon flux for IKNO in the UV range when the ring is operating in the ultra-stable CSR mode with 3 ps natural bunch length. 
In a more exotic scheme by slicing the beam when the current in the bunch is above the MBI threshold, it is possible to seed and control the instability onset. In this situation the instability gain can be exploited to significantly increase the power of the radiated CSR (Heifets \& Stupakov, 2006; Byrd et al., 2006, Phys Rev. Lett. 97).

\section{The IKNO Laboratory.}

IKNO is also the name of the Italian infrastructure, proposed in Sardinia to host this new high performance electron storage ring. The project for the facility (Curedda \& Pusceddu, 2007) has been submitted to the Italian Roadmap of the Research Infrastructure (https://roadmap.miur.cineca.it/). The IKNO complex is designed to be completely autonomous and includes offices, preparation laboratories and everything else required for the operation of the facility and assistance to the users. Figure 5 shows a top view of the storage ring building of the IKNO facility. The storage ring area is clearly visible together with a number of possible beamlines and experimental areas located on the plane of the ring. The figure also shows the tunnel designed to accommodate the linear accelerator (linac) used for the injection of the electrons in the ring at the energy of operation. The linac solution allows for a more flexible injection scheme but is more expensive with respect to the "classical" scheme using a lower energy small linac in combination with a "booster" ring that accelerates the electron to the operation energy before the injection in the storage ring. The decision on what injection configuration to use is still under evaluation.

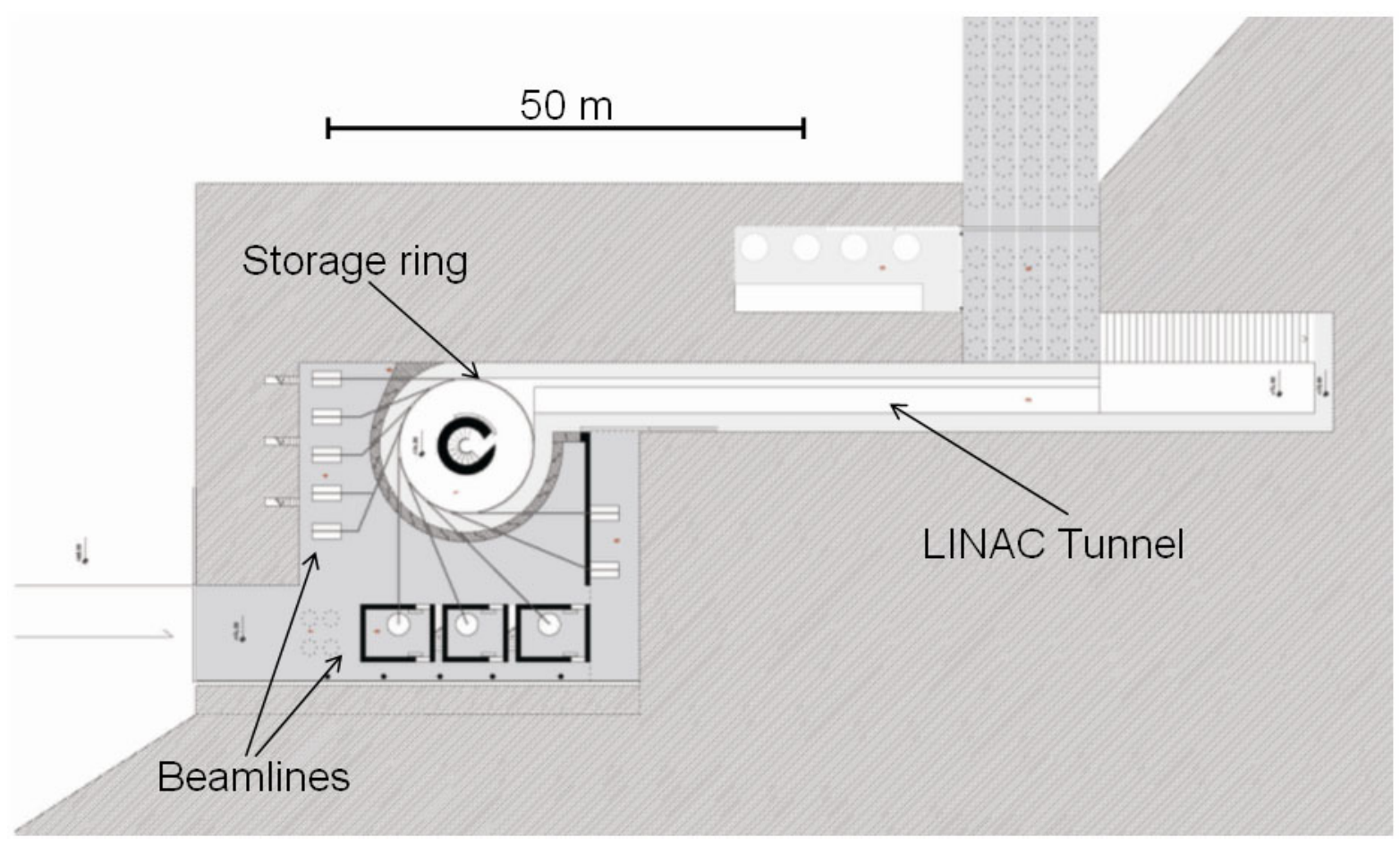

Figure 5 Top view of a possible layout for the storage ring building. The figure shows the tunnel for the linac (the linear accelerator used for injecting the beam into the storage ring), the storage ring hall, and some of the beamlines and experimental areas in the storage ring plane. Also shown in the figure, some of the laboratories and of the structures required for the operation of the facility. 


\section{Conclusions.}

IKNO is a proposal for a SR facility to be built in Sardinia, Italy, which includes a storage ring-based source optimised for the generation of CSR in the terahertz frequency range. This under-exploited region of the electromagnetic spectrum offers research opportunities that only high-brightness large-scale facilities may allow to reach. The revolutionary performance of IKNO in terms of photon flux offers opportunities in physics, chemistry, biology, medicine, materials science and opens to new exciting research opportunities in space applications, imaging, engineering and homeland security. In addition and simultaneously to the terahertz CSR generation, IKNO also produces an incoherent photon flux extending up to the VUV frequencies and comparable with the one of existing 3rd generation light sources. IKNO is a real multi-user facility that accommodates a large number of bending magnets beamlines plus several insertion devices for further extending and improving the performance of the source.

\section{References}

Abo-Bakr, M., Feikes, J., Holldack, K., Wüstefeld, G. \& Hübers, H.-W. (2002). Phys. Rev. Lett. 88, 254801.

Abo-Bakr, M., Feikes, J., Holldack, K., Kuske, P. \& Wüstefeld, G. (2003). Proceedings of the 2003 Particle Accelerator Conference, Portland, OR USA, 3023-3025.

Abo-Bakr, M., Feikes, J., Holldack, K., Kuske, P., Peatman, W. B., Schade, U., Wüstefeld, G. \& Hübers, H.-W. (2003), Phys.

Rev. Lett. 90, 094801.

Bane, C., Krinsky S. \& Murphy J. B. (1995). AIP Proc. 367, 191-199.

Barry, W. C., et al. (2002). Proceedings of the 2002 European Particle Accelerator Particle Accelerator EPAC02, La Villette, Paris, 656-658.

Bielawski, S., Evain, C., Hara, T. Hosaka, M., Katoh, M., Kimura, S., Mochihashi, A., Shimada, M., Szwaj, C., Takahashi, T. \& Takashima, Y (2008). Nature Physics 4, 390-393.

Byrd, J. M., Leemans, W. P., Loftsdottir, A., Marcelis, B., Martin, M. C., McKinney, W. R., Sannibale, F., Scarvie, T. \& Steier, C. (2002). Phys. Rev. Lett. 89, 224801.

Byrd, J. M., Martin, M. C., McKinney, W. R., Munson, D. V., Nishimura, H., Robin, D. S., Sannibale, F., Schlueter, R. D., Thur, W. G., Jung, J. Y. \& Wan, W. (2004). Infrared Phys. \& Tech. 45, 325-330.

Byrd, J.M., Hao, Z., Martin, M. C., Robin, D.S., Sannibale, F., Schoenlein, R.W., Zholents, A.A. \& Zolotorev., M.S. (2005). Proceedings of the 2005 Particle Accelerator Conference, Knoxville, TN, 3682-3684.

Byrd, J. M., Martin, M. C. \& Sannibale, F. (2005). CERN Courier, Vol. 45, 7, 39-42.

Byrd, J. M., Hao, Z., Martin, M. C., Robin, D. S., Sannibale, F., Schoenlein, R. W., Zholents, A. A. \& Zolotorev, M. S. (2006). Phys. Rev. Lett. 96, 164801.

Byrd, J. M., Hao, Z., Martin, M. C., Robin, D. S., Sannibale, F., Schoenlein, R. W., Zholents, A. A. \& Zolotorev, M. S. (2006). Phys. Rev. Lett. 97, 074802.

Carr, G. L., Martin, M. C., McKinney, W. R., Jordan, K., Neil, G. R. \& Williams, G. P. (2002). Nature 420, $153-156$. Curedda, D. \& Pusceddu F. (2007). Laurea Thesis, "IKNO: Una proposta di grande infrastruttura scientifica in Sardegna", Sassari University. 
De Santis, S., Byrd, J. M., Li, D. \& Sannibale, F. (2003). "Longitudinal Impedance Measurements of the CIRCE Arc Vacuum Chamber", CIRCE Note LSCN-IF-1. Lawrence Berkeley National Laboratory, Berkeley, CA USA.

Heifets, S. \& Stupakov, G. (2006). SLAC-PUB-11815.

Holldack, K., Kachel, T., Khan, S., Mitzner, R. \& Quast., T. (2005). Phys. Rev. ST-AB 8, 040704.

Holldack, K., Khan, S., Mitzner, R. \& Quast., T. (2006). Phys. Rev. Lett. 96, 054801.

Murphy, J. B., Krinsky, S. \& Gluckstern, R. (1997). Particle Accelerators 57, 9-21.

Nishimura, H., Robin, D., Sannibale, F. \& Wan, W. (2004). Proceedings of the 2004 European Particle Accelerator Conference, Lucerne, Switzerland, 2182-2184.

Sannibale, F., Byrd, J. M., Loftsdóttir, Á., Venturini, M., Abo-Bakr, M., Feikes, J., Holldack, K., Kuske, P., Wüstefeld, G., Hübers, H.-W., Warnock, R. (2004). Phys. Rev. Lett. 93, 094801.

Sannibale, F., Byrd, J. M., Loftsdóttir, Á., Venturini, M., Abo-Bakr, M., Feikes, J., Holldack, K., Kuske, P., Wüstefeld, G., Hübers, H.-W. \& Warnock, R. (2004). ICFA Beam Dynamics Newsletter 35, 27-39.

Shen, Y., Watanabe, T., Arena, D. A., Kao, C.-C., Murphy, J. B., Tsang, T. Y., Wang, X. J. \& Carr, G. L. (2007). Phys. Rev.

Lett. 99, 043901.

Sherwin, M. S., Schmuttenmaer, C. A., \& Bucksbaum, P. H. (2004). "Opportunities in THz Science", DOE-NSF-NIH Workshop, Arlington, VA, USA, February 2004 (http://www.sc.doe.gov/bes/reports/files/THz_rpt.pdf).

Stupakov G. \& Heifets S. (2002). Phys. Rev., ST Accel. Beams 5, 054402.

Venturini, M. \& Warnock, R. (2002). Phys. Rev. Lett. 89, 224802.

Zholents, A. A. \& Zolotorev, M. S. (1996). Phys. Rev. Lett. 76, 912. 